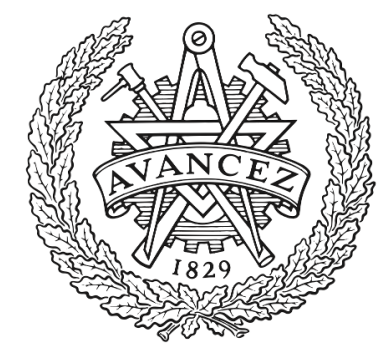

CHALMERS

UNIVERSITY OF TECHNOLOGY

\title{
Radar Interference Mitigation through Active Coordination
}

Downloaded from: https://research.chalmers.se, 2023-04-26 13:28 UTC

Citation for the original published paper (version of record):

Aydogdu, C., Keskin, F., Carvajal, G. et al (2021). Radar Interference Mitigation through Active Coordination. IEEE National Radar Conference - Proceedings, 2021-May.

http://dx.doi.org/10.1109/RadarConf2147009.2021.9455180

N.B. When citing this work, cite the original published paper. 


\title{
Radar Interference Mitigation through Active Coordination
}

\author{
Canan Aydogdu*, Musa Furkan Keskin*, Gisela K. Carvajal ${ }^{\dagger}$, Olof Eriksson ${ }^{\ddagger}$, Hans Hellsten ${ }^{\S}$, Hans Herbertsson ${ }^{\ddagger}$, \\ Emil Nilsson ", Mats Rydström ${ }^{\ddagger}$ Karl Vanäs", Mustafa Mete*, Per Sandrup ", Henk Wymeersch* \\ ${ }^{*}$ Chalmers University of Technology, Sweden, ${ }^{\dagger}$ QAMCOM Research, Sweden, ${ }^{\ddagger}$ Veoneer, Sweden \\ ${ }^{\S} \mathrm{SAAB}$, Sweden ${ }^{\top}$ Halmstad University, Sweden, "Volvo Car Corporation, Sweden
}

\begin{abstract}
Intelligent transportation is heavily reliant on radar, which have unique robustness under heavy $\mathrm{rain} / \mathrm{fog} / \mathrm{snow}$ and poor light conditions. With the rapid increase of the number of radars used on modern vehicles, most operating in the same frequency band, the risk of radar interference becomes an important issue. As in radio communication, interference can be mitigated through coordination. We present and evaluate two approaches for radar interference coordination, one for FMCW and one for OFDM, and highlight their challenges and opportunities.
\end{abstract}

\section{INTRODUCTION AND MOTIVATION}

All wireless transmission systems are subject to interference. This is because the propagation medium, i.e., the frequency band in the wireless channel, is shared among all active transmitters. The problem of interference in radar (see Fig. 1) is more serious than in communication for two distinct reasons: first of all, the interfering signal in radar can be a one-way signal, while the radar signal of interest is a twoway signal. This makes the interfering signal much stronger than the desired radar signal and thus hard to mitigate in post-processing. Secondly, radar functionality is often related to safety, so that intermittent radar interference can lead to missed targets (e.g., pedestrians are not properly detected due to a strong noise over all Dopplers or ranges) or false alarms (e.g., the vehicle suddenly breaks because interference causes a ghost target). Nevertheless, current automotive radars are unlikely to get issues noticeable to the customer, the mutual interference problem is expected to become more challenging, unless properly handled [1], as new vehicles are equipped with a larger number of radars providing unparalleled awareness at short, medium, and long ranges [2]. To address this foreseen problem, intense research and development activities have been conducted in the past decade [3], [4].

Interference-free operation will require radar transmission standardization. A standardized transmission scheduling system resembling today's mobile communication system would present a solution to the interference problem, but it is not without challenges. The most common automotive radar is frequency modulated continuous wave (FMCW) radar. FMCW waveforms can, up to a point, relatively easily be repaired in case it is intermittently corrupted by interference [5], which is why they are still operational. On the other hand, current and future communication systems have also gone up in frequency,

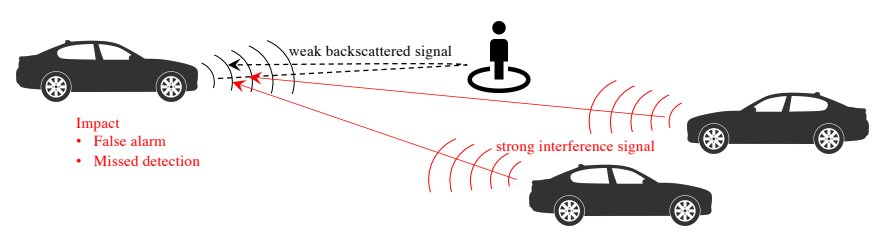

Fig. 1. Interference is generally much stronger than the desired radar signal, due to the one-way propagation. Interference increases with more interfering radars and leads to false alarms and missed detections.

enabling re-use of $5 \mathrm{G}$ and beyond $5 \mathrm{G}$ waveforms for radar and sensing applications [6], [7]. The most widely used communication waveform is orthogonal frequency division multiplexing (OFDM), which has been extensively studied for radar purposes [8], [9]. OFDM has the added benefit that radarrelated data can be shared to improve situational awareness and safety. A comparison of FMCW and full-band OFDM radar under interference is presented in [10], whereas the focus in this article is spectrally efficient OFDM.

In this paper, we consider the FMCW and OFDM radar waveforms and present recent results related to their coexistence capabilities. The contributions of this work are as follows: (i) For both FMCW and OFDM, we provide a qualitative interference analysis; (ii) For FMCW, we present interference mitigation results of the RadChat protocol [11], based on real traffic data from [12]; and (iii) For OFDM, we present range-Doppler profiles of several spectrally efficient waveforms to assess their radar capabilities and demonstrate their performance based on experimental data.

\section{RADAR MODELS AND QUALITATIVE INTERFERENCE ANALYSIS}

\section{A. FMCW Radar}

The transmit waveform of an FMCW radar with $K$ consecutive linear frequency modulated (LFM) chirp\$ 1 (or sweeps) can be expressed as [13] $s(t)=\sum_{k=0}^{K-1} x(t-k T)$, where the individual chirps are given by

$$
x(t)=e^{j \varphi(t)}, \varphi(t)=2 \pi\left(f_{c} t+0.5 \alpha t^{2}\right),
$$

\footnotetext{
${ }^{1}$ Here, we assume no idle periods between consecutive chirps. It is also possible to introduce random inter-chirp idle periods to reduce the probability of mutual interference between FMCW radars.
} 
for $0 \leq t \leq T$. Here, $\alpha=B / T$ is the chirp slope, $B$ denotes the sweep bandwidth, $T$ represents the chirp duration and $f_{c}$ is the carrier frequency. Suppose there exists a single target of interest acting as a point scatterer, characterized by a complex channel gain $\gamma$ (including the effects of path loss, antenna gain and radar cross section), an (initial) round-trip propagation delay $\tau=2 R / c$ and a normalized relative Doppler shift $\nu=$ $2 v / c$, where $R$ and $v$ denote, respectively, the distance and relative radial velocity between the radar and target, and $c$ is the speed of wave propagation. The received backscattered signal is of the form (stop-and-hop assumption [14, Ch. 2.6.2])

$$
r_{k}(t)=\gamma x(t+(t+k T) \nu-\tau)+w_{k}(t)
$$

where $0 \leq t \leq T$ denotes the time relative to the beginning of the $k$ th chirp, where $w_{k}(t)$ is measurement noise. After dechirping, low-pass filtering with bandwidth $B_{\mathrm{s}}=\tau_{\max } \alpha$, sampling with a period of $T_{s}$ for $\tau_{\max } \leq t \leq T$ ( $\tau_{\max }$ is the maximum round-trip delay), we rearrange into a slow-timefast-time data matrix, where the $k$ th row contains the samples of the $k$ th chirp (fast time), while the $n$th column contains the $n$th sample of each chirp (slow time). In other words,

$$
y_{k, n}=\gamma e^{j 2 \pi\left(-\alpha \tau+f_{c} \nu\right) n T_{s}} e^{j 2 \pi f_{c} \nu k T}+w_{k, n}
$$

for $k=0, \ldots, K-1$ and $n=n_{\max }, \ldots, N-1, n_{\max }=$ $\left\lfloor\tau_{\max } / T_{s}\right\rfloor, N=\left\lfloor T / T_{s}\right\rfloor+1$, and $w_{k, n}$ are independent and identically distributed (i.i.d.) complex Gaussian noise samples with variance $\sigma^{2}$. A 2D discrete Fourier transform (DFT) can be applied across slow-time and fast-time dimensions of the beat signal in (3), which yields the FMCW delay-Doppler spectrum, with peak value proportional to the processing gain $G_{\mathrm{p}}=K\left(N-n_{\max }\right)$. The peaks of the delay-Doppler spectrum provide range and Doppler estimates of the target.

FMCW Interference: Suppose an interfering radar employs an FMCW waveform with chirp slope $\widetilde{\alpha}=\widetilde{B} / \widetilde{T}$, sweep bandwidth $\widetilde{B}$ and chirp duration $\widetilde{T}$. The samples (3) then become

$$
y_{k, n}=\gamma e^{j 2 \pi\left(-\alpha \tau+f_{c} \nu\right) n T_{s}} e^{j 2 \pi f_{c} \nu k T}+\gamma_{\mathrm{int}} x_{\mathrm{int}, k, n}+w_{k, n} .
$$

Interference is generally much stronger than the desired backscattered signal, as they are governed by the Friis free space propagation equation and the radar equation respectively [15]:

$$
\left|\gamma_{\mathrm{int}}\right|^{2}=P \frac{G_{\mathrm{trx}} \lambda^{2}}{(4 \pi)^{2} r^{2}}, \quad|\gamma|^{2}=P \frac{G_{\mathrm{trx}} \sigma \lambda^{2}}{(4 \pi)^{3} d^{4}},
$$

where $r$ is the distance between the interferer and the victim radar, $d$ is the distance between the radar and the target, $P$ is the transmit power, $G_{\text {trx }}$ is the combined transmit and receive antenna gain, $\sigma$ is the radar cross section of the target. Hence, for similar $d$ and $r$ and typical values of $\sigma,\left|\gamma_{\text {int }}\right|^{2} \gg|\gamma|^{2}$. The nature of the interference depends on the total interference power (i.e., the aggregate power of the interference samples), and the level of coherence between victim and interfering radar [16], [17]. To illustrate how a ghost target is spread out depending on the relative waveform parameters, Fig. 2 2 shows the fast time FFT output, i.e., the range FFT, corresponding to an interfering radar signal as a function of distance. The larger

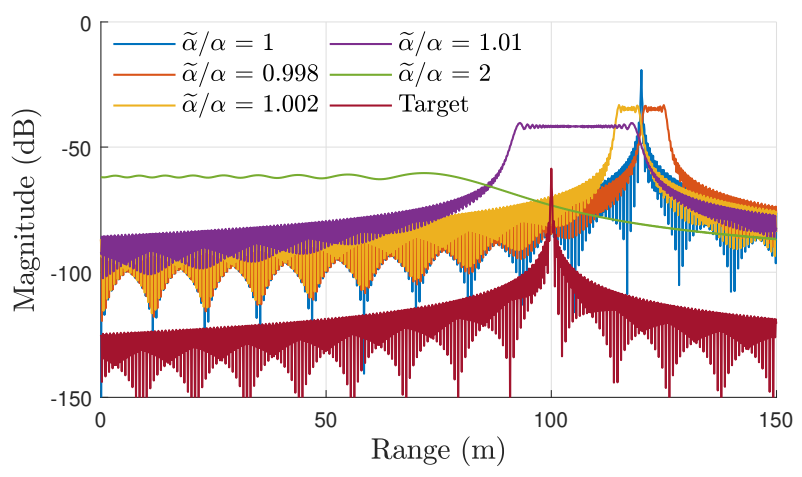

Fig. 2. FMCW range profiles in the presence of an interfering radar for various values of chirp slope ratios, with $f_{c}=77 \mathrm{GHz}, B=1 \mathrm{GHz}, T=20 \mu \mathrm{s}$.

the difference in the chirp slope, the more the interference is spread out, due to the decrease in coherence. This affects the detection of targets in various ways. Incoherent interference may hinder the detection of low RCS targets (pedestrians, cyclists) over a large fraction of the delay-Doppler domain, whereas a (partially) coherent interference can mask even high RCS targets (vehicles) but in a smaller fraction of the delayDoppler domain.

\section{B. OFDM Radar}

In this type of radar, the waveform used is an OFDM signal known from communications. The processing details are documented in [9] and are summarized here. The transmit signal consists of parallel orthogonal subcarriers, each modulated with a data. The resulting baseband time-domain signal for the $k$-th OFDM symbol is expressed as [8]

$$
x(t)=\sum_{n=0}^{N-1} d_{k, n} e^{j 2 \pi n \Delta f t}, k T \leq t \leq(k+1) T,
$$

for $k=0, \ldots, K-1$, where $N$ represents the number of subcarriers, $K$ is the number of consecutive symbols evaluated, $\Delta f$ is the subcarrier spacing, $T=T_{\mathrm{cp}}+T_{\mathrm{sym}}$ is the OFDM symbol duration consisting of the cyclic prefix (CP) duration $T_{\mathrm{cp}}$ and the elementary symbol duration $T_{\mathrm{sym}}$, and $d_{n, k}$ represents the complex modulation symbol (the arbitrary data modulated with a discrete phase modulation technique, e.g., quadrature amplitude modulation (QAM)) on subcarrier $n$, OFDM symbol $k$. Note that not all subcarriers need to be used, so that $d_{k, n}=0$ is also possible. We will assume that $\left|d_{k, n}\right| \in\{0,1\}$, to lighten the presentation.

The processing of the backscattered signal consists of the following steps [9, Ch. 3.2]: (i) removal of the $\mathrm{CH}^{2}$, (ii) Fourier transform over the elementary symbol duration, and (iii) element-wise complex division by the transmit symbols (called reciprocal filtering in OFDM radar processing [18]).

\footnotetext{
${ }^{2}$ The CP duration is assumed to be larger than the round-trip time of the furthermost target $[9$. Ch. 3.2.1]. In contrast to OFDM communications, OFDM radar is mono-static and does not require synchronization to detect the $\mathrm{CP}$ interval of the incoming signal.
} 
Then, for the $n$th subcarrier of the $k$ th OFDM symbol, we obtain

$$
y_{k, n}=\gamma e^{-j 2 \pi n \Delta f \tau} e^{j 2 \pi f_{c} \nu k T}+z_{k, n} .
$$

Similar to FMCW processing, taking the two-dimensional Fourier transform over frequency and time dimensions in (6) yields estimates of range and Doppler parameters. Let $\boldsymbol{D} \in \mathbb{R}^{K \times N}$ describe the time-frequency allocation, with $[\boldsymbol{D}]_{k, n}=\left|d_{k, n}\right| \in\{0,1\}$. The processing gain is then $G_{\mathrm{p}}=\|\boldsymbol{D}\|_{F}^{2}$, i.e., the squared Frobenius norm of $\boldsymbol{D}$.

OFDM Interference: Consider, for simplicity, an interfering OFDM radar with data symbols $\tilde{d}_{k, n}$. The observation $y_{k, n}$ then becomes

$$
y_{k, n}=\gamma e^{-j 2 \pi n \Delta f \tau} e^{j 2 \pi f_{c} \nu k T}+\gamma_{\mathrm{int}} \frac{\tilde{d}_{k, n}}{d_{k, n}}+z_{k, n} .
$$

Note that there is only interference for those symbols and subcarriers where $\tilde{d}_{k, n} d_{k, n} \neq 0$. Hence, the power allocation across time and subcarriers is crucial in OFDM radar. The signal power in the target bin is $P_{S}=|\gamma|^{2}\|\boldsymbol{D}\|_{F}^{4}$, the noise power is $P_{N}=N_{0}\|\boldsymbol{D}\|_{F}^{2}$, and the interference power is

$$
P_{I}=\left|\gamma_{\text {int }}\right|^{2}\|\boldsymbol{D} \odot \tilde{\boldsymbol{D}}\|_{F}^{2},
$$

where $\odot$ denotes the pointwise multiplication. Hence, the overlap in time-frequency should be sufficiently small to ensure that $P_{I}<P_{S}$. In OFDM communication, a similar problem arises, where $\|\boldsymbol{D} \odot \tilde{\boldsymbol{D}}\|_{F}^{2}=0$ is guaranteed by orthogonal time-frequency allocation and scheduling. Assuming a duty cycle of $u \leq 1$ for OFDM radar transmission, the maximum number of radars that can be active simultaneously, while still satisfying $\|\boldsymbol{D} \odot \tilde{D}\|_{F}^{2}=0$ is bounded by

$$
N_{\max }=\left\lfloor\frac{K N}{\|\boldsymbol{D}\|_{F}^{2} u}\right\rfloor .
$$

\section{Active CoORdinAtion}

The impact of interference includes ghost targets and increases in noise floor. Both are detrimental to radar operations. Approaches to deal with interference can be grouped as either reactive, which aim to reduce the impact of interference after it has occurred, or proactive, which aims to avoid or reduce interference by design. The current attitude toward interference mitigation in the industry focuses on various techniques, including pulse to pulse processing and removing polluted pulses, sniffing and avoiding used frequencies, cognitive approaches [19], using frequency diversity, using narrow main beams or side-lobe null steering [3]. Other reactive strategies exploit sparsity of useful signal and interference components [20], [21]. Interference avoidance techniques can also be more invasive, such as notifying the driver, disabling the sensor feature, shifting function to another sensor, reducing CFAR detection sensitivity [3]. However, these avoidance mechanisms either decrease the radar detection performance or disable the radar completely [22].

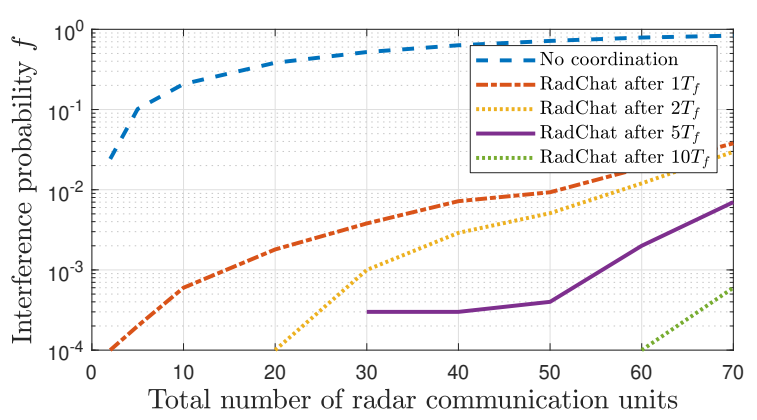

Fig. 3. Radar interference probability $f$ after one frame time $\left(T_{f}=20 \mathrm{~ms}\right)$, $2 T_{f}=40 \mathrm{~ms}, 5 T_{f}=100 \mathrm{~ms}, 10 T_{f}=1 \mathrm{~s}$ with coordinated transmission for varying number of radars for 10,000 Monte-Carlo simulations, $B=1 \mathrm{GHz}$, $B_{\mathrm{s}}=50 \mathrm{MHz}, T=20 \mu s, f_{c}=79 \mathrm{GHz}, K=99$ with contention window size of 6 .

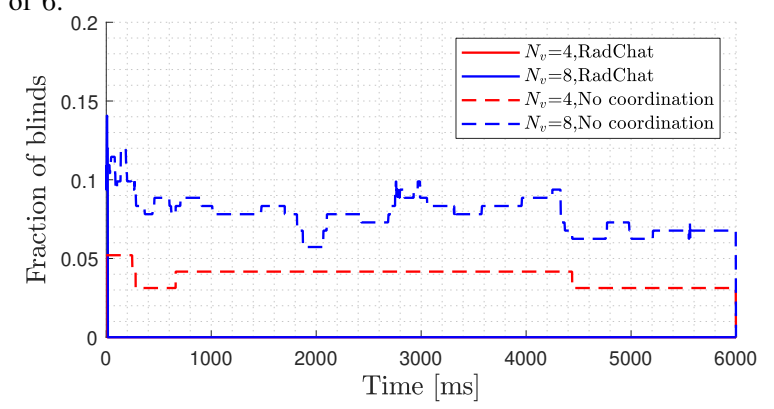

Fig. 4. Fraction of blind with and without coordination for a real traffic scenario with number of vehicles $N_{v}=\{4,8\}, B=250 \mathrm{MHz}$ for corner radars and $B=800 \mathrm{MHz}$ for front and back-end radars, $B_{\mathrm{s}}=15 \mathrm{MHz}$, $T_{f}=50 \mathrm{~ms}, T=20 \mu \mathrm{s}, f_{c}=79 \mathrm{GHz}, K=128$ with contention window size of 48 .

\section{A. Active coordination in FMCW - RadChat}

Coordination of automotive radars through communications and assigning radars disjoint frequency-time-space resources is another option to mitigate interference. This is possible since current radars inefficiently use the frequency band (radars receive over a small fraction $B_{\mathrm{s}} / B$ of the available bandwidth), time (radars are genrally active with low duty cycles) and space (radar field of view is directional and signals are blocked mostly by other vehicles). The resource assignment requires coordination through distributed network communication so that it functions safely in case of inavailability of a cellular infrastructure. This distributed communication should also be low-latency due to the highly dynamic vehicular networks, which can be achieved by upgrading automotive radars to joint radar communication units (RCU). RCUs exploit the similarity of the radar circuitry to standard communication hardware, use the same hardware for both radar and communications and are composed of data link and physical layers. Communication occurs over a fixed and dedicated communication bandwidth, which is limited by the ADC. The radar and communication functionality are time-multiplexed for duty cycles 3 of $u<1$. We introduces RadChat as a distributed cooperation-based radar communication protocol in [11] for the coordination of RCUs, which uses these principles.

${ }^{3}$ When $u \approx 1$, the benefit of deploying RCUs becomes negligible and 802.11p or C-V2X are more appropriate. 


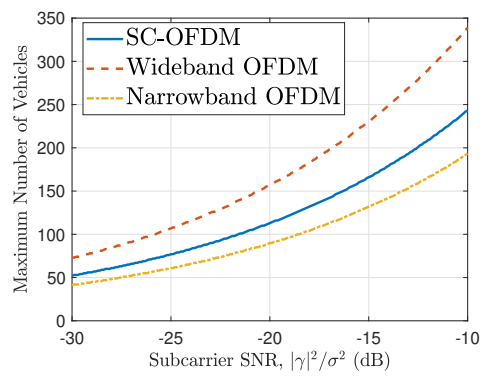

(a)

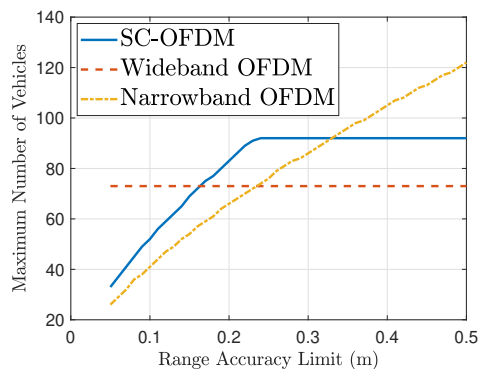

(b)

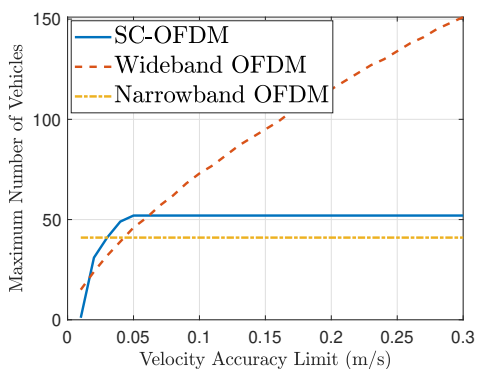

(c)

Fig. 5. Maximum number of vehicles that can be accommodated within a time-frequency resource defined by $B_{\text {tot }}=1 \mathrm{GHz}$ and $T_{\text {tot }}=30 \mathrm{~ms}$ for the three different OFDM waveforms, where $f_{0}=77 \mathrm{GHz}, \Delta f=500 \mathrm{KHz}, T_{\mathrm{cp}}=400 \mathrm{~ns}$. Stepped OFDM and narrowband OFDM schemes require an ADC with $50 \mathrm{MHz}$ rate, whereas wideband OFDM requires $1 \mathrm{GHz}$-rate ADC. (a) With respect to subcarrier SNR, where range and velocity accuracy limits are $0.1 \mathrm{~m}$ and $0.1 \mathrm{~m} / \mathrm{s}$, respectively. (b) With respect to range accuracy limit, where subcarrier SNR is $-30 \mathrm{~dB}$ and velocity accuracy limit is $0.1 \mathrm{~m} / \mathrm{s}$. (c) With respect to velocity accuracy limit, where subcarrier SNR is $-30 \mathrm{~dB}$ and range accuracy limit is $0.1 \mathrm{~m}$.

Fig. 3 shows the interference probability (i.e., the expected value of $f$ ) versus the number of radars, with and without coordination for a vehicular network, where all radars are assumed to be in FoV of all the other radars. The propagation of the coordination information to the whole vehicular network for a distributed protocol is time-dependent, hence after each radar frame time $T_{f}$, the interference probability drops significantly for varying number of radars.

A real traffic data set, which consists of the trace of vehicular traffic in Cologne [12], is used to evaluate the impact of RadChat on the coordination of the radars. Since this vehicular mobility data is taken by intervals of a minute for twenty-four hours, it is interpolated by $10 \mu \mathrm{s}$ by assuming vehicular movement as a continuous function. Vehicles are assumed to be equipped with four medium range corner radars, one front and one back-end long range radar. The fraction of blinds (i.e., the ratio of interference-blinded RCUs to the total number of RCUs) for the real traffic data set, which is obtained over a $6 \mathrm{~s}$ interval for a total of 10 Monte-Carlo simulations (given in Fig. 4), shows that the coordination of radars mitigates interference in the first $11.4 \mathrm{~ms}$ and $15 \mathrm{~ms}$ when RadChat is applied. However, without coordination, the ratio of the number of blinded radars varies around $5 \%$ and 10 $\%$ for changing number of vehicles $N_{v} 4$ and 8 , respectively.

\section{B. Active coordination in OFDM-Orthogonal time-frequency allocation}

OFDM has also been studied extensively as a radar waveform ${ }^{4}[8],[9],[18],[24]$ A way around this problem is the use of Stepped-Carrier OFDM (SC-OFDM) scheme, where consecutive OFDM symbols consisting of a certain number of contiguous subcarriers are transmitted at different carrier frequencies to cover a large overall bandwidth [25], [26].

\footnotetext{
${ }^{4}$ Under certain conditions, OFDM and FMCW radars with identical waveform parameters exhibit similar performance in terms of accuracy and resolution. OFDM provides the additional capability of communications at the expense of increased hardware complexity due to OFDM modulator/demodulator operations [23].
}

Time-frequency resource management coordinated by a central unit (e.g., a 5G base station) can reduce interference among radars on different vehicles, similar to conventional OFDM radar networks [9, Ch. 4], which requires synchronization between the central node and vehicles. In [27], vehicle-to-vehicle $(\mathrm{V} 2 \mathrm{~V})$ sidelink resource allocation is investigated for SC-OFDM joint radar-communication networks, demonstrating trade-offs between range/velocity accuracy and data rate. Fig. 5 shows exemplary results for three different OFDM strategies 5 namely, stepped-carrier, narrowband, and wideband, where in the latter case each vehicle uses the total bandwidth (and thus requires an ADC with $1 \mathrm{GHz}$ sampling rate) for a duration $K T$ and then remains silent for a duration $(M-1) K T$. Here, $K$ is the number of consecutive OFDM symbols and $M$ is the number of different carrier frequencies used by SC-OFDM. As seen from the figures, the SC-OFDM radar can support more vehicles in a given spectral resource than the conventional narrowband OFDM radar with the same ADC rate since the former offers the flexibility to trade off a decrease in Doppler accuracy for an improvement in ranging accuracy (frequency hopping enhances ranging accuracy and decreases Doppler accuracy). In Fig. 5(b), as wideband OFDM is mainly restricted by the velocity accuracy constraint, relaxing the range accuracy constraint does not further improve its performance (similarly, for narrowband OFDM in Fig. 5(c)).

Fig. 6 illustrates the resource allocation for a vehicle in a given time-frequency grid for the SC-OFDM scheme and the resulting range and velocity profiles obtained as the zero-Doppler and zero-delay slices of the waveform ambiguity function $(A F)^{6}$. To increase the spectral efficiency further, other alternative waveforms can be considered, such as stepped-carrier stepped-time OFDM (SCST-OFDM) and continuous carrier randomized OFDM (CCR-OFDM), as shown

\footnotetext{
${ }^{5}$ As a measure of range/velocity accuracy, we use the theoretical bounds from 27 .

${ }^{6}$ Here, the periodic AF [28] of the OFDM waveform is derived, where the processing is performed over the entire time-frequency region of interest by setting $d_{k, n}=0$ for the unused subcarriers in (5).
} 


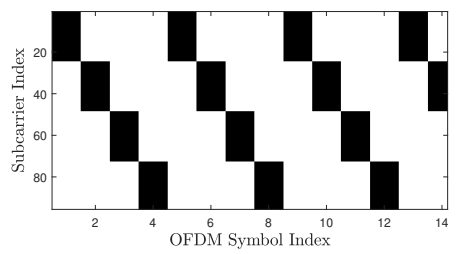

(a)

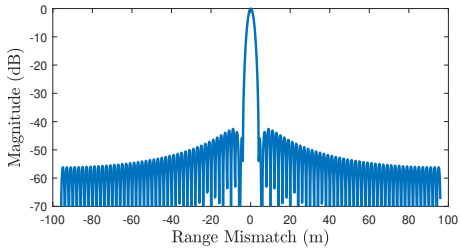

(b)

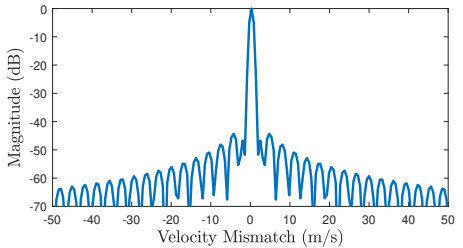

(c)

Fig. 6. (a) SC-OFDM time-frequency allocation $D$ with total bandwidth $B=150 \mathrm{MHz}, N=96$ subcarriers, 24 contiguous subcarriers and $K=4576$ symbols, (b) range profile of SC-OFDM waveform, and (c) velocity profile of SC-OFDM waveform.

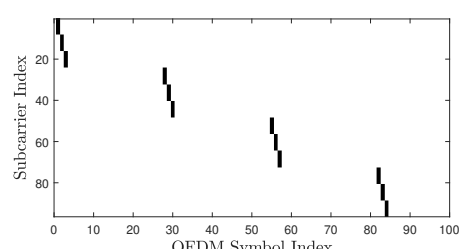

(a)

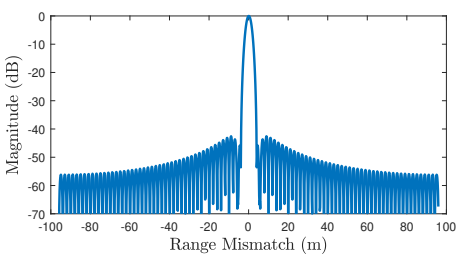

(b)

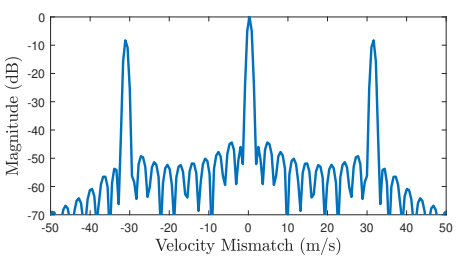

(c)

Fig. 7. (a) SCST-OFDM time-frequency allocation $D$ with total bandwidth $B=150 \mathrm{MHz}, N=96$ subcarriers, 8 contiguous subcarriers, $K=4576$ symbols and 3 symbols per block, (b) range profile of SCST-OFDM waveform, and (c) velocity profile of SCST-OFDM waveform.

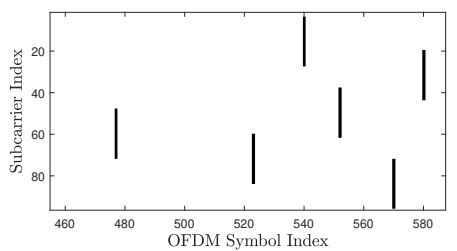

(a)

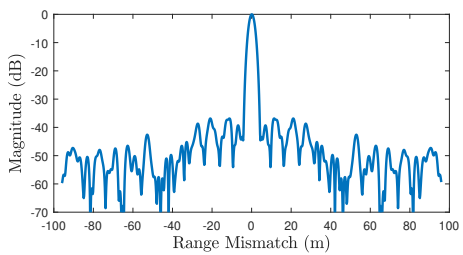

(b)

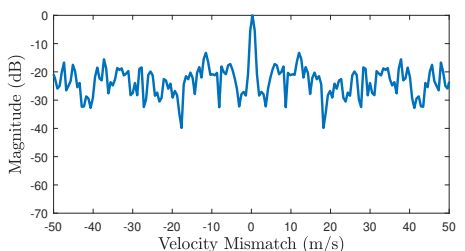

(c)

Fig. 8. (a) CCR-OFDM time-frequency allocation $D$ with total bandwidth $B=150 \mathrm{MHz}, N=96$ subcarriers, 24 contiguous subcarriers and $K=4576$ symbols, (b) range profile of CCR-OFDM waveform, and (c) velocity profile of CCR-OFDM waveform.

in Fig. 7 and Fig. 8, respectively. In SCST-OFDM, there are gaps in both time and frequency between consecutive symbol blocks to accommodate more vehicles. On the other hand, the CCR-OFDM scheme relies on a random multiplexing idea [29] and allocates at most one block of non-overlapping subcarriers (with the starting subcarrier selected uniformly at random) in each OFDM symbol according to a Bernoulli distribution. The secondary peaks in the velocity profile of the SCST-OFDM waveform result from the periodic gaps in the time domain (i.e., idle subcarriers). Since a certain number of contiguous subcarriers are utilized in the frequency domain (i.e., transmission blocks are contiguous in frequency, but not in time), the range side-lobes of the SCST-OFDM waveform have significantly less power.

Our demonstrator platform, complete with millimeter-wave front-ends, high speed digital signal generation and signal acquisition, and independent generation of arbitrary interference, has been used to assess several of the considered OFDM waveforms. The communication bandwidth is $150 \mathrm{MHz}$, using $N=96$ subcarriers at a carrier of $60 \mathrm{GHz}$. A total of $K=4576$ OFDM symbols were transmitted with QPSK data. Four waveforms were compared: wideband OFDM using all 


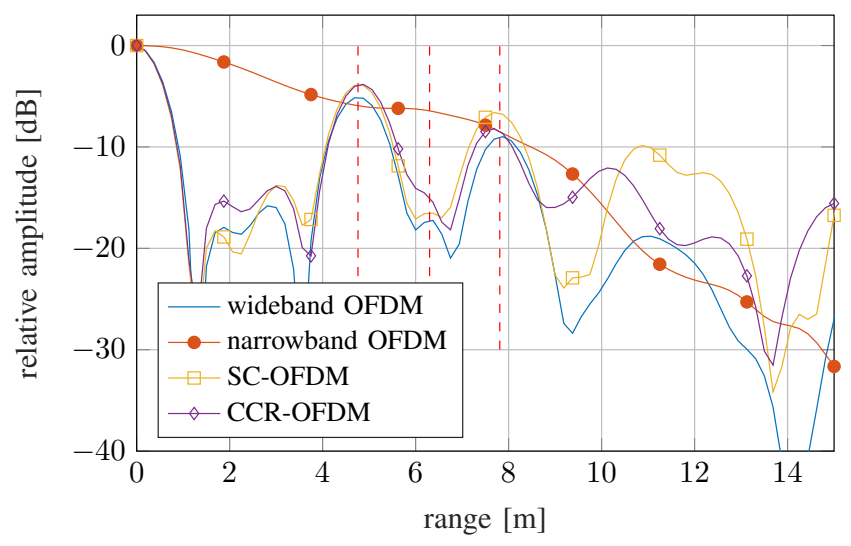

Fig. 9. Results with demonstrator for different OFDM waveform alternatives The environment contained three targets (a reflector at $4.8 \mathrm{~m}$, a wall at 6.3 $\mathrm{m}$, and corner at $7.8 \mathrm{~m}$ ).

96 subcarriers, narrowband OFDM (using 24 fixed consecutive subcarriers), SC-OFDM (using a time-varying window of 24 consecutive subcarriers), CCR (using a random window of 24 consecutive subcarriers). From the results in Fig. 9 we observe that the experimental results largely confirm the theory, with SC-OFDM and CCR-OFDM exhibiting side-lobes, while narrowband OFDM fails to detect any of the targets.

\section{CONCLUSION}

FMCW radar interference is expected to be an increasing problem as more vehicles are equipped with more radars. While FMCW has inherent robustness to interference and standard reactive approaches are expected to be sufficient for the coming years, long-term solutions require standardization. In this paper, we have evaluated two strategies for active radar interference mitigation: RadChat and spectrally efficient OFDM. Each of these has specific challenges, either in terms of signal processing, channel access, or resource allocation. We show that RadChat can remove FMCW interference, provided radars can be synchronized. On the other hand, the selected OFDM waveforms were demonstrated to lead to good radar performance, though with potentially large sidelobes, and also come with a need for synchronization (on the order of a fraction of an OFDM symbol).

\section{ACKNOWLEDGMENTS}

This research is supported by Vinnova grant 2018-01929.

\section{REFERENCES}

[1] H. Meinel and J. Dickmann, "Automotive radar: From its origin to future directions," Microwave Journal, vol. 56, pp. 24-40, Sep. 2013.

[2] C. Aydogdu, M. F. Keskin et al., "Radar interference mitigation for automated driving: Exploring proactive strategies," IEEE Signal Processing Magazine, vol. 37, no. 4, pp. 72-84, 2020.

[3] W.Buller, B. Wilson et al., "Radar congestion study," (Report No. DOT HS 812 632). Washington, DC: National Highway Traffic Safety Administration, Tech. Rep., Sep. 2018.

[4] I. M. Kunert, "Project final report, MOSARIM: More safety for all by radar interference mitigation," 2012. [Online]. Available: http://cordis.europa.eu/docs/projects/cnect/1/248231/ 080/deliverables/001-D611finalreportfinal.pdf
[5] J. Moss and A. Lefevre, "Radar interference suppression," 2019-05-29 (pub), patent Application EP 3489710.

[6] C. B. Barneto, T. Riihonen et al., "Full-duplex OFDM radar with LTE and 5G NR waveforms: Challenges, solutions, and measurements," IEEE Transactions on Microwave Theory and Techniques, vol. 67, no. 10, pp. 4042-4054, 2019.

[7] A. Bourdoux, A. N. Barreto et al., "6G white paper on localization and sensing," arXiv preprint:2006.01779, 2020.

[8] C. Sturm and W. Wiesbeck, "Waveform design and signal processing aspects for fusion of wireless communications and radar sensing," Proceedings of the IEEE, vol. 99, no. 7, pp. 1236-1259, July 2011.

[9] M. Braun, "OFDM radar algorithms in mobile communication networks," Karlsruher Institutes für Technologie, 2014.

[10] G. K. Carvajal, M. F. Keskin et al., "Comparison of automotive fmcw and ofdm radar under interference," in 2020 IEEE Radar Conference (RadarConf20), 2020, pp. 1-6.

[11] C. Aydogdu, M. F. Keskin et al., "Radchat: Spectrum sharing for automotive radar interference mitigation," IEEE Transactions on Intelligent Transportation Systems, vol. 22, no. 1, pp. 416-429, 2021.

[12] S. Uppoor, O. Trullols-Cruces et al., "Generation and analysis of a largescale urban vehicular mobility dataset," IEEE Transactions on Mobile Computing, vol. 13, no. 5, pp. 1061-1075, 2014.

[13] S. M. Patole, M. Torlak et al., "Automotive radars: A review of signal processing techniques," IEEE Signal Processing Magazine, vol. 34, no. 2, pp. 22-35, 2017.

[14] M. A. Richards, Fundamentals of Radar Signal Processing. Tata McGraw-Hill Education, 2005.

[15] A. Al-Hourani, R. J. Evans et al., "Stochastic geometry methods for modeling automotive radar interference," IEEE Transactions on Intelligent Transportation Systems, vol. 19, no. 2, pp. 333-344, 2017.

[16] M. Goppelt, H.-L. Blöcher, and W. Menzel, "Automotive radarinvestigation of mutual interference mechanisms," Advances in Radio Science, vol. 8, no. B. 3, pp. 55-60, 2010.

[17] G. Kim, J. Mun, and J. Lee, "A peer-to-peer interference analysis for automotive chirp sequence radars," IEEE Transactions on Vehicular Technology, vol. 67, no. 9, pp. 8110-8117, Sep. 2018.

[18] S. Mercier, S. Bidon et al., "Comparison of correlation-based OFDM radar receivers," IEEE Transactions on Aerospace and Electronic Systems, 2020.

[19] Z. Slavik and K. V. Mishra, "Cognitive interference mitigation in automotive radars," in 2019 IEEE Radar Conference (RadarConf), April 2019 , pp. $1-6$

[20] F. Uysal, "Synchronous and asynchronous radar interference mitigation,' IEEE Access, vol. 7, pp. 5846-5852, 2019.

[21] J. Bechter, F. Roos et al., "Automotive radar interference mitigation using a sparse sampling approach," in European Radar Conference (EURAD), Oct 2017, pp. 90-93.

[22] S. Alland, W. Stark et al., "Interference in automotive radar systems: Characteristics, mitigation techniques, and current and future research," IEEE Signal Processing Magazine, vol. 36, no. 5, pp. 45-59, Sep. 2019.

[23] J. Fink and F. K. Jondral, "Comparison of OFDM radar and chirp sequence radar," in IEEE International Radar Symposium (IRS), 2015, pp. $315-320$.

[24] C. R. Berger, B. Demissie et al., "Signal processing for passive radar using OFDM waveforms," IEEE Journal of Selected Topics in Signal Processing, vol. 4, no. 1, pp. 226-238, 2010.

[25] G. Lellouch, A. K. Mishra, and M. Inggs, "Stepped OFDM radar technique to resolve range and doppler simultaneously," IEEE Transactions on Aerospace and Electronic Systems, vol. 51, no. 2, pp. 937-950, April 2015.

[26] B. Schweizer, C. Knill et al., "Stepped-carrier OFDM-radar processing scheme to retrieve high-resolution range-velocity profile at low sampling rate," IEEE Transactions on Microwave Theory and Techniques, vol. 66 , no. 3, pp. 1610-1618, March 2018.

[27] M. F. Keskin, C. Aydogdu, and H. Wymeersch, "Stepped-carrier OFDM V2V resource allocation for sensing and communication convergence," in 14th European Conference on Antennas and Propagation, 2020.

[28] R. F. Tigrek, W. J. A. De Heij, and P. Van Genderen, "OFDM signals as the radar waveform to solve doppler ambiguity," IEEE Transactions on Aerospace and Electronic Systems, vol. 48, no. 1, pp. 130-143, Jan 2012.

[29] C. Knill, F. Roos et al., "Random multiplexing for an MIMO-OFDM radar with compressed sensing-based reconstruction," IEEE Microwave and Wireless Components Letters, vol. 29, no. 4, pp. 300-302, 2019. 